\title{
Effect of pravastatin on biliary lipid composition and bile acid synthesis in familial hypercholesterolaemia
}

\author{
N Hoogerbrugge-vd Linden, F W M de Rooy, H Jansen, $M$ van Blankenstein
}

\begin{abstract}
Nine patients with heterozygous familial hypercholesterolaemia were treated for eight weeks with either $\mathbf{4 0} \mathbf{~ m g}$ pravastatin or placebo under double blind conditions. Six patients received pravastatin, a competitive inhibitor of 3-hydroxy-3-methylglutary1-coenzyme A (HMG-CoA) reductase. Treatment with pravastatin resulted in a significant decrease in plasma cholesterol caused by a decrease in low density lipoprotein cholesterol (LDL-c) of $30 \%(p<0 \cdot 005)$. We determined the effect of this medication on the lithogenicity of bile. Cholesterol saturation index of fasting gall bladder bile decreased with $23 \%$ ( $p<0.01$ ) from 1.06 to 0.75 during treatment with pravastatin. A reduction of $24 \%(p<0.01)$ in molar percentage of biliary cholesterol was seen. After treatment the total bile acid excretion in faeces and the molar percentage of biliary bile acids were not significantly changed, suggesting that pravastatin does not influence bile acid biosynthesis to a significant extent. These findings indicate that treatment with pravastatin can decrease the incidence and complications of cholesterol gall stones.
\end{abstract}

Pravastatin (eptastatin, CS-514, SQ $31 \cdot 000$ ), is a new hypocholesterolaemic agent which effectively lowers total plasma cholesterol, by competitive inhibition of 3-hydroxy-3-methylglutaryl-coenzyme A (HMG-CoA) reductase, the rate limiting enzyme in cholesterol synthesis. ${ }^{12}$ Next to lowering plasma cholesterol this drug can also influence biliary cholesterol excretion. ${ }^{3}$ A reduction in cholesterol synthesis can theoretically lead to changes in bile acid synthesis, resulting in unpredictable changes in bile composition. Supersaturation of bile with cholesterol predisposes to formation of gall stones. ${ }^{4}$ Other systemic lipid lowering drugs, like clofibrate and gemfibrozil increase cholesterol saturation of bile, thereby enhancing the risk of gall stone formation. ${ }^{56}$ Data on the effect of HMG-CoA reductase inhibitors on bile composition in animals are limited and give conflicting results. Tsujita showed that pravastatin induces a reduction of the cholesterol saturation index (CSI) in dogs by decreasing the biliary neutral sterols and cholesterol, without effects on phospholipids and bile acids. ${ }^{7}$ In another study Kempen et al found in rats that compactin, another HMGCoA reductase inhibitor, resulted in a decrease of bile cholesterol. In that study, however, bile acid secretion decreased as well. ${ }^{8}$ Duane et al recently ${ }^{9}$ found that treatment with simvastatin of 10 patients with non-familial hypercholesterolaemia induced a decrease in mean cholesterol saturation index. The present investigation was designed to determine whether patients treated with pravastatin because of familial hypercholesterolaemia, are at risk for developing cholesterol gall stones.

\section{Methods}

\section{SUBJECTS}

Nine patients (two postmenopausal women and seven men), aged 22-54 years, with heterozygous familial hypercholesterolaemia gave informed consent and participated in this study. Heterozygous familial hypercholesterolemia was diagnosed by family history, tendon xanthomata and plasma lipid analysis. The mean of two terminations of fasting LDL-cholesterol concentrations obtained at least one week apart was more than $5.0 \mathrm{mmol} / \mathrm{l}$ in all patients. The mean plasma triglyceride concentration in the same specimens was less than $4.0 \mathrm{mmol} / \mathrm{l}$. Drugs known to affect plasma lipid levels were prohibited during the study. Treatment with thiazide diuretics and beta-blockers was continued and kept constant from eight weeks before randomisation throughout the study. Patients with diabetes mellitus, hypothyroidism or any other cause of secondary hyperlipidaemia were excluded from the study. The plasma lipid concentration was stabilised for at least eight weeks on a lipid lowering diet. In the last four weeks of this stabilisation period all patients were given placebo. After this period the patients were randomised and given either $40 \mathrm{mg}$ pravastatin or placebo under double blind conditions. Medication was taken before retiring to bed. Six patients received pravastatin, three placebo. Compliance was checked by counting tablets. The study was approved by the Medical Ethics Committee of the University Hospital Rotterdam.

\section{Sample Collection}

Fasting blood samples and bile were collected before and after eight weeks of treatment. Blood samples were collected after an overnight fast. Plasma cholesterol and triglyceride concentrations were determined by enzymatic methods (Boehringer-test kit combination). Lipoprotein fractions were separated after 24 hours ultracentrifugation of the plasma at $40.000 \mathrm{rpm}$ at $4^{\circ} \mathrm{C}$ using a SW-41 rotor in a Beckmann L5-50 UC. ${ }^{10}$

Gall bladder bile was collected after an overnight fast through duodenal intubation. The tip of the intubation tube was placed before the ampulla of Vater as judged by gastroscopy and controlled by $x$ ray examinations. Gall bladder contraction was induced by im injection of 0.3 $\mu \mathrm{g} / \mathrm{kg}$ bodyweight of ceruletide (Takus, 
TABLE I Plasma lipid concentrations before $(B)$ and after $(A)$ treatment for eight weeks of nine patients with familial hypercholesterolaemia, six patients were treated with $40 \mathrm{mg}$ pravastatin daily, three with placebo

\begin{tabular}{|c|c|c|c|c|}
\hline & Cholesterol & $L D L-c$ & $H D L-c$ & $T G$ \\
\hline $\begin{array}{l}\text { Treated patients } \\
\text { B } \\
\text { A } \\
\text { p-value: }\end{array}$ & $\begin{array}{l}10 \cdot 3(2 \cdot 2) \\
7 \cdot 5(1 \cdot 1) \\
<0.01\end{array}$ & $\begin{array}{l}7 \cdot 4(1 \cdot 7) \\
5 \cdot 1(1 \cdot 0) \\
<0.01\end{array}$ & $\begin{array}{l}1.09(0.38) \\
1 \cdot 14(0.34) \\
\text { NS }\end{array}$ & $\begin{array}{l}1.59(1.02) \\
1.46(0.51) \\
\text { NS }\end{array}$ \\
\hline $\begin{array}{l}\text { Placebo treated patie } \\
\text { B } \\
\text { A }\end{array}$ & $\begin{array}{l}\text { ts } \\
9 \cdot 8(1 \cdot 5) \\
9 \cdot 4(1 \cdot 4)\end{array}$ & $\begin{array}{l}7 \cdot 2(0 \cdot 2) \\
6 \cdot 7(0 \cdot 2)\end{array}$ & $\begin{array}{l}1.11(0.32) \\
0.94(0.44)\end{array}$ & $\begin{array}{l}1.99(2.0) \\
1.58(1.0)\end{array}$ \\
\hline
\end{tabular}

Data are presented as mean (standard deviation) and expressed in $\mathrm{mmol} / \mathrm{l}$ ).

Farmitalia). After collection, the gall bladder bile was diluted 1:9 with isopropanol and centrifuged for 10 minutes at $1300 \mathrm{~g}$. The supernatant was used for determination of phospholipid concentration." Cholesterol was determined in the supernatant by an enzymatic method (Boehringer-test kit combination). Faecal bile acid excretion was quantified as bile acids deconjugated by alkaline hydrolysis, in the extract of homogenised four days stool collection. Stools were collected after swallowing 25 radioopaque markers. For each patient the distribution pattern and the amount of markers found in the collected faeces before and after treatment was not significantly different. Bile acid concentration in the isopropanol extract of the gall bladder bile as well as in the faeces extract was determined using the 3hydroxysteroid dehydrogenase method adapted from Bruusgaard, ${ }^{12}$ (Sterognost-Flu, Nyegaard, Norway). The cholesterol saturation index was calculated according to Carey. ${ }^{13}$

\section{CLINICAL FOLLOW UP OF PATIENTS}

Physical examination was performed every second week. In addition electrocardiographic and ophthalmologic examinations were performed before and after the observation period. Haematologic studies, clinical chemistry profiles and prothrombin time were done every second week.

TABLE II Gall bladder bile composition, cholesterol saturation index (CSI) and faecal bile acid loss before $(B)$ and after $(A)$ treatment for eight weeks of nine patients with familial hypercholesterolaemia, six patients were treated with $40 \mathrm{mg}$ pravastatin daily, three with placebo. Gall bladder bile composition is expressed as molar percentage

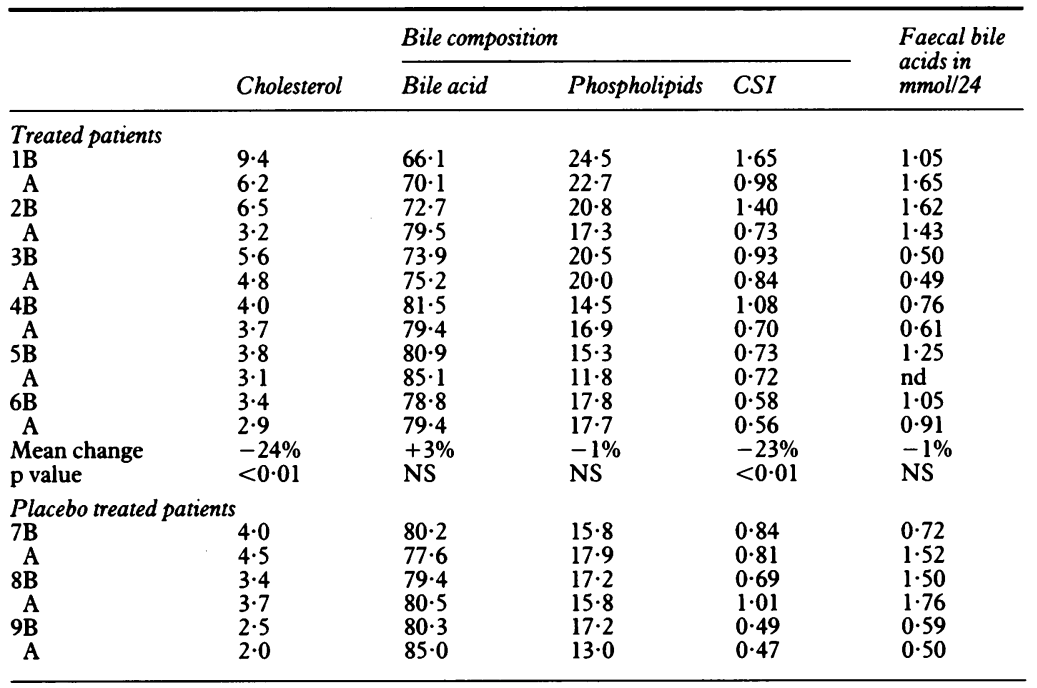

STATISTICAL ANALYSIS

The Wilcoxon's rank-sum test was used for comparisons within the treated groups before and after treatment.

\section{Results}

\section{EFFECTS OF PRAVASTATIN ON}

\section{LIPOPROTEIN CONCENTRATIONS}

After treatment with pravastatin for eight weeks a significant decrease of total plasma cholesterol of $24 \%(p<0.01)$ was seen, which was caused by a lowering in low density lipoprotein cholesterol of $30 \%(p<0.005)$ as illustrated in Table I. In contrast total plasma triglycerides and high density lipoprotein cholesterol concentrations were not significantly changed. The placebo treated patients did not show any significant change in lipoprotein concentrations.

\section{EFFECTS OF PRAVASTATIN ON} COMPOSITION OF GALL BLADDER BILE

The gall bladder bile of six patients treated with pravastatin, showed a significant decrease in cholesterol saturation index from 1.06 to 0.75 $(23 \%, \mathrm{p}<0.01)$ (Figure). The most impressive decreases in cholesterol saturation index $(48,40$ and $35 \%$ ) were seen in three patients having indexes above 1.0 before therapy (indicating an increased risk for gall stone formation). In faeces total bile acid excretion was not influenced by pravastatin treatment (Table II). In bile the molar percentage of bile acids was not changed either, while the molar percentage of biliary cholesterol was significantly reduced by $24 \%$ $(p<0.001)$. As expected the concentration of phospholipids did not change. The placebo treated patient did not show any effect on either the gall bladder bile composition, or the faecal bile acid excretion (Table II). None of the patients showed any side effect.

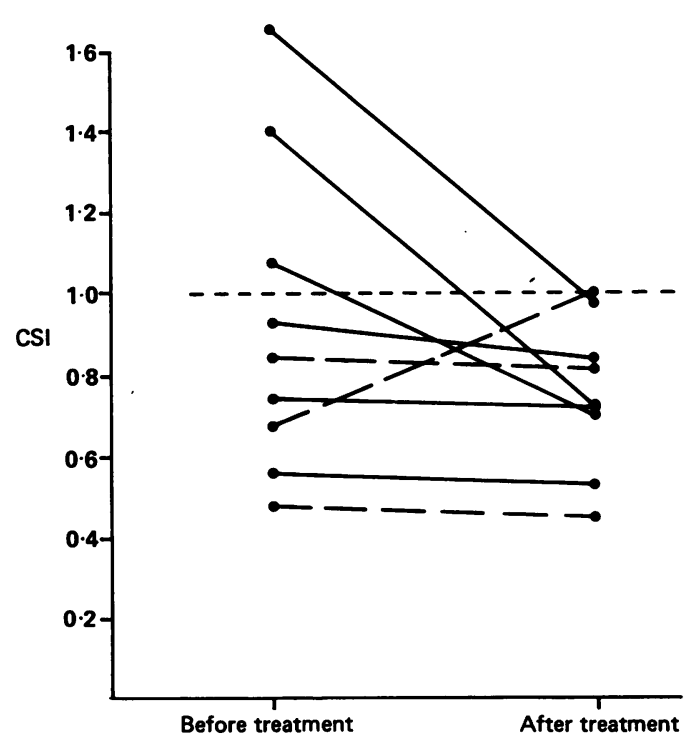

Figure: Cholesterol saturation index (CSI) of gall bladder bile before and after treatment for eight weeks of nine patients with familial hypercholesterolaemia, six patients were treated
with $40 \mathrm{mg}$ pravastatin (closed lines), three with placebo (dotted lines). 


\section{Discussion}

Six patients with familial hypercholesterolaemia were treated with $40 \mathrm{mg}$ pravastatin and showed a significant decrease in plasma cholesterol concentration as a result of a decrease in low density lipoprotein cholesterol concentration. In addition to the effect on plasma cholesterol, we found a significant decrease in cholesterol saturation index of fasting gall bladder bile after treatment with pravastatin. In a steady state the faecal bile acid excretion practically equals total hepatic bile acid synthesis. In our pravastatin treated patients, the faecal bile acid excretion and the molar percentage of biliary bile acids were not significantly changed. This suggests that pravastatin did not alter total bile acid synthesis to a great extent. In contrast, we did see a reduction in molar percentage of biliary cholesterol. The combination of a steady bile acid excretion and a decreased cholesterol saturation index indicates that the altered bile composition can be a result of a decreased biliary cholesterol excretion. Grundy ${ }^{3}$ showed a reduction in cholesterol excretion in some familial hypercholesterolaemia patients treated with mevinolin.

The decreases in cholesterol saturation index induced by pravastatin are comparable with those after treatment with chenodeoxycholic acid or ursodeoxycholic acid. ${ }^{14}$ Perhaps a combination of pravastatin with those older drugs can even have a more pronounced effect. Our data suggest that pravastatin (and probably other HMG-CoA reductase inhibitors as well) can decrease the incidence of cholesterol gall stones and gall stone complications. If this effect on cholesterol saturation index is confirmed in larger studies of longer duration, HMG-CoA reductase inhibiton may prove valuable in the medical treatment and prevention of patients with recurrent cholesterol gall stones.
The authors thank Prof J H P Wilson for his valuable suggestions. Pravastatin was kindly provided by SQUIBB, Rijswijk (ZH), The Netherlands

1 Nakaya N, Homma Y, Tamachi H, Shigematsu H, Hata Y, Goto Y. The effect of CS-514 on serum lipids and Apolipoprotein in hypercholesterolemic subjects. FAMA 1987; 237 : 3083-93.

2 Yoshino G, Kazumi T, Iwai M, et al. Effect of CS-514 on plasma lipids and lipoprotein composition in hyperplasma lipids and lipoprotein composition in hyper-

3 Grundy SM, Bilheimer DW. Inhibition of 3-hydroxy-3 methylglutaryl-CoA reductase by mevinolin in familial methylglutaryl-CoA reductase by mevinolin in familial hypercholesterolemia heterozygotes: effects on cholest

4 Grundy SM, Metzger AL, Adler Rd. Mechanisms of lithogenic bile formation in American Indian women with cholesterol bile formation in American Indian women

5 Leiss O, von Bergmann K, Gnasso A, et al. Effect of gemfibrozil on biliary lipid metabolism in normolipemic subjects. Metabolism 1985; 34: 74-82.

6 Pertsemlidis D, Panveliwaila D, Ahrens E J Jr. Effects of clofibrate and an estrogen-progestagen combination on fasting biliary lipids and cholic acid kinetics in man. Gastroenterology 1974; 66: 565-73.

7 Tsujito Y, Kulogy 17 , 66: $565-73$. inhibitor of 3-hydroxy-3-methylglutaryl Coenzyme A reductase: tissue-selective inhibition of sterol synthesis and reductase: tissue-selective inhibition of sterol synthesis and
hypolipidemic effect on various animal species. Biochim hypolipidemic effect on vario
Biophys Acta 1986;877: $50-60$.

8 Kempen HJM, de Lange J, Vos-van Holstein MPM, Van Wachem P, Havinga R, Vonk RJ. Effect of ML-236 B (compactin) on biliary excretion of bile salts and lipids, and on bile flow, in the rat. Biochim Biophys Acta 1984; 794: 43543.

9 Duane WC, Huuninghake DB, Freeman ML, Pooler PA, Schlasner LA, Gebhard RL. Simvastatin, a competitive inhibitor of HMG-CoA reductase, lowers cholesterol saturation index of gall bladder bile. Hepatology 1988; 8: 1147-50.

10 Redgrave TG, Robets DCK, West CE. Separation of plasma lipoproteins by density gradient ultracentrifugation. Anal lipoproteins by density

11 Goodwin JF, Thibert R, McCann D, Boyle AJ. Estimation of serum phospholipide and total phosphorus using choloric acid. Anal Chem 1958; 30: 1097-9.

12 Bruusgaard A. Quantitative determination of the major 3hydroxy bile acids in biological material after thin-layer chromatographic separation. Clin Chim Acta 1970; 28: 495504

13 Carey MC. Critical tables for calculating the cholesterol saturation of native bile. 7 Lipid Res 1978; 19: 945-55.

14 Tint GS, Salen G, Shefer S. Effect of ursodeoxycholic acid and chenodeoxycholic acid on cholesterol and bile acid metabolism. Gastroenterology 1986; 91: 1007-18. 